\title{
Correction to: Ayahuasca as a Candidate Therapy for PTSD
}

\author{
Jessica L. Nielson and Julie D. Megler
}

\section{Correction to:}

Chapter 3 in: B. Caiuby Labate and C. Cavnar (eds.), The Therapeutic Use of Ayahuasca, https://doi.org/10.1007/978-3-642-40426-9_3

In the original version of this book, the following correction has been incorporated: In Chapter 3, the name citation of "John Pasquina" has been removed. The book and the chapter have been updated with the change. 